\title{
LES PSYCHOTHERAPIES BREVES PSYCHODYNAMIQUES ET RELATIONNELLES ET LEUR EMPLOI ACTUEL DANS LE TRAITEMENT DES DYSFONCTIONS SEXUELLES
}

\author{
Jean Tignol,
}

Université de Bordeaux II - Centre Carreire - 121, rue de la Béchade - 33076 BORDEAUX CEDEX

\section{BRIEF PSYCHODYNAMIC OR INTER- PERSONAL PSYCHOTHERAPY IN THE TREATMENT OF SEXUAL DISORDERS.}

Brief psychodynamic or interpersonal therapeutic techniques, which were developed mainly in the UK and USA, are either derived from psychoanalysis or are closely related to it. The use of standardized methods during their development allowed them to be used in research protocols in the same way as biological treatments. Consequently, their general efficacity was established during the 1980s based upon reliable data. These techniques' foundation upon common underlying mechanisms of action allowed the use of several psychotherapeutic techniques either within a given patient or by an individual therapist. Subsequently, generalized psychotherapeutic models based on the various methods were elaborated, resulting in the widespread applicability of these techniques. In the treatment of sexual disorders brief psychotherapeutic techniques now address more complex intrapsychic factors or interpersonal problems involved in sexual dysfunction than did the sex therapy models of Masters and Johnson and their early followers. Two types of situations can be distinguished where the indications are either symptomatic or non-symptomatic, although such a dichotomy may be too strict. The latter category is more of an individual situation where attention is not concentrated on the sexual symptoms but deals more with the patient's general problems. The former category deals mainly with treating a couple's secondary sexual disorder problem(s) where attention is focussed on the symptoms and is often associated with prescribing behavioural tasks. Debate over the use of intracavernosal injections in association with psychotherapy continues. In conclusion, the efficacy of dynamic and interpersonal psychotherapeutic techniques has now been established; although their use in the field of sexual disorders must be complementary, not concurrent, to biological treatments. This is an area of continued scientific research. Key words : Brief psychotherapy, psychodynamic therapy, interpersonal therapy, sexual disorders. Andrologie, 1992, $2:$ 137-140.

\section{DEFINITIONS}

Par psychothérapie, nous entendons ici une psychothérapie réglée, c'est-à-dire qui implique une technique précise, en général soustendue par une théorie explicite. Il ne s'agira donc pas du tout dans notre exposé de l'effet psychothérapique général de la relation inter-humaine, très important dans la relation médecin/malade, et qui peut être alors soit méconnu, soit utilisé de façon empirique et/ou implicite.

On s'accorde pour appeler psychothérapie brève une psychothérapie qui comprend au maximum vingt séances.

La définition d'une psychothérapie psychodynamique et/ou relationnelle est plus large. On désigne par là un ensemble de psychothérapies inspirées par la psychanalyse ou proches d'elle. Si une de ces psychothérapies réglées met plutôt l'accent sur la dynamique intrapsychique on la qualifiera plutôt de psychodynamique ; si au contraire c'est la dynamique interpersonnelle qui est privilégiée, on la qualifiera plutôt de relationnelle.

\section{HISTORIQUE}

Un bref historique est nécessaire pour situer ce courant psychothérapique vis-à-vis de la psychanalyse qui en est la source.

Freud déjà avait émis l'idée qu'une certaine activité du thérapeute pourrait peut-être accélérer le processus psychanalytique. C'est son élève Férenczi qui mit en pratique cette idée; mais ses efforts dans ce sens furent ensuite désavoués par Freud qui trouva son élève un peu trop actif. Par la suite ce balancement entre le désir d'accélérer le processus psychanalytique et les "dangers" des procédures utilisées pour cela va se reproduire à plusieurs reprises. Il aboutira au développement des psychothérapies brèves en Angleterre et aux Etats-Unis, alors que les psychanalystes français ne s'y intéresseront pratiquement plus à partir des années 60.
Il faut toutefois signaler l'existence d'un précurseur français, René Held (3), tant en matière de psychothérapies brèves d'inspiration psychanalytique que de leurs applications au traitement des troubles sexuels. Dès 1956, cet auteur a prononcé une conférence sur la psychothérapie de l'impuissance sexuelle, publiée en 1957. Il a aussi présenté au Congrès de Psychanalystes de Langue Romane de 1963 un rapport sur les psychothérapies d'inspiration psychanalytique. Ces deux textes ont été réunis avec quelques autres dans son livre "Psychothérapie et Psychanalyse" paru en 1968. Ses écrits ont été très précieux pour moi lorsque j'ai débuté dans le traitement psychothérapique des troubles sexuels en 1972.

L'historique des psychothérapies brèves proprement dites est très bien décrit dans l'article de Gillieron (5). Cet auteur cite les précurseurs et créateurs : Lewin 1970, Mann 1973, Malan 1976, Sifneos 1977, Davanlo 1978. Il existe entre les techniques proposées par ces différents auteurs des variations qui portent sur le degré plus ou moins important d'activité du thérapeute et sur la durée du traitement. Gillieron souligne que plus le dispositif spatio-temporel est clairement déterminé, plus le thérapeute parait pouvoir laisser le processus se dérouler librement, tandis qu'à l'inverse lorsque le dispositif spatiotemporel est plus flou, le thérapeute se montre plus actif et même extrêmement actif ou éclectique. Gilliéron (4) est le seul auteur francophone important, mais il est suisse, de Lausanne, et non francais.

\section{EVOLUTION ACTUELLE}

L'évolution des psychothérapies brèves psychodynamiques et relationnelles s'est faite, de la fin des années 70 au début des années 80 , vers la réalisation de psychothérapies standardisées et expérimentables.

Cet effort de standardisation s'est traduit par l'apparition de manuels dont les plus connus concernent l'Inter Personnal Therapy (I.P.T) de Klerman (9), psychothérapie relationnelle, et la Supportive Expressive Therapy (S.E.T) de Luborsky (12), psychothérapie psychodynamique. L'année de parution de ces manuels est 1984. Chacun précise les bases théoriques de la 
psychothérapie, la technique, les procédures de formation des thérapeutes et les procédures d'évaluation.

Ces psychothérapies brèves deviennent ainsi expérimentables, c'est-à-dire peuvent être mises à l'épreuve de recherches scientifiques visant à leur évaluation, similaires à celles utilisées pour les traitements biologiques. On peut obtenir sur leurs indications et leurs résultats des données fiables par des procédures satisfaisant aux exigences méthodologiques de la recherche clinique. C'est ainsi que l'I.P.T a pu être comparée à une psychothérapie cognitivo-comportementale, à l'imipramine, et à un placebo dans la grande étude collaborative du N.I.M.H sur le traitement de la dépression (2).

Orlinsky (14) dans son article "Apports de la recherche à la connaissance des psychothérapies", expose les données actuelles de la recherche, dont les suivantes:

- Tout d'abord certaines techniques, telles que donner des conseils, sont toujours inefficaces. $\mathrm{La}$ sagesse populaire savait bien que "les conseilleurs ne sont pas les payeurs" ; désormais la sagesse psychothérapique le sait aussi.

Par contre l'efficacité des psychothérapies réglées est maintenant démontrée par les métaanalyses qui combinent quantitativement les effets observés dans de nombreuses enquêtes distinctes. L'amélioration qu'entraîne la psychothérapie est proportionnelle à la dose de psychothérapie, mais il y a plus de progrès au début que par la suite. Les patients qui restent en psychothérapie continuent à s'améliorer, mais la vitesse de leur progression se ralentit au fur et à mesure que la psychothérapie s'allonge.

Par ailleurs et de facon apparemment paradoxale, les études comparatives n'ont pas démontré la supériorité d'une quelconque approche psychothérapique sur une autre. C'est donc que les psychothérapies ne tirent pas leur efficacité de leur théorie ou de leur méthodologie qui sont différentes, mais de processus communs sous-jacents. Ceci a conduit divers auteurs à proposer un modèle générique de psychothérapie, modèle qui peut s'appliquer à toutes les psychothérapies et rendre ainsi compte de leurs effets.

Celui d'Orlinsky (13) est très intéressant et très complet, mais à cause de cela la place nous manque pour l'étudier en détail. Il souligne, après d'autres, l'importance du lien relationnel psychothérapique, qui agit par lui-même comme une intervention psychothérapique, et qui influence fortement les autres paramètres du processus psychothérapique. Sous le nom d'alliance thérapeutique, ce lien relationnel a fait l'objet de nombreuses recherches sur son rôle dans les psychothérapies, avec entre autres la construction d'échelles pour sa mesure (6).

Un dernier trait important de l'évolution actuelle des psychothérapies brèves est l'apparition de l'éclectisme en psychothérapie qui se justifie par l'existence de facteurs communs aux différentes psychothérapies et de modèles génériques. L'utilisation éclectique des psychothérapies permet de reconnaître des utilisations préférentielles à certaines psychothérapies. Elle permet aussi l'association de procédures appartenant à des techniques différentes dans la psychothérapie d'un sujet donné. L'éclectisme en psychothérapie est un mouvement complexe qui peut bien sûr pêcher par "trop d'éclectisme", et aussi dans lequel se poursuit la lutte territoriale des différents mouvements psychothérapiques. Toutefois cet éclectisme a fait preuve d'une efficacité importante dans certains domaines : par exemple les troubles anxieux et aussi le sujet qui nous occupe aujourd'hui, les troubles sexuels.

\section{LES PSYCHOTHERAPIES PSYCHODYNAMIOUES ET RELATIONNELLES DANS LE TRAITEMENT DES TROUBLES SEXUELS : ETAT ACTUEL}

On peut dire aujourd'hui que grâce aux efforts des psychothérapeutes américains, ces thérapies résistent et même regagnent du terrain, vis-à-vis de la tendance expansionniste actuelle des thérapies cognitivo-comportementales.

On peut, je crois, faire remonter les sexothérapies modernes à Masters et Johnson (15). Ces auteurs ont, avec un succès extraordinaire, promu l'abord direct du symtôme sexuel par des méthodes psychothérapiques de type comportemental, bien qu'euxmêmes ne se réfèrent pas explicitement au comportementalisme. Cette réhabilitation de l'abord direct du symptôme et du corps, qui se révèle très efficace, va donner lieu à quelques excès d'activisme militant dans la ligne idéologique de la sexualité libérée et libératrice. Dès 1975 toutefois, on revient à une conception plus psychodynamique de la sexothérapie, dont le travail d'Helen Kaplan (7), aux U.S.A, peut être tenu pour représentatif.

Actuellement toutefois, ces divers modèles semblent dépassés et on pourrait parler avec Lo Piccolo (11) de sexothérapies "postmodernes".

Comme dans les courants artistiques dits postmodernes, la créativité de chacun est libérée sans référence obligatoire à une tendance dominante, et les thérapeutes éclectiques peuvent ainsi associer comportementalisme et/ou cognitivisme, et/ou thérapies d'inspiration systémique, et/ou thérapies d'inspiration psychodynamique, et/ou relationnelles. Une autre caractéristique commune de ces sexothérapies postmodernes est la prise en considération du caractère adaptatif du symptôme, prise en considération que l'on trouve aussi bien chez Lo Piccolo qui est un cognitivo-comportementaliste ayant intégré plus tard des principes systémiques, que chez Althof (1) qui est un thérapeute psychodynamique utilisant des prescriptions comportementales.

Enfin, les modèles de dysfonctionnement et les modèles thérapeutiques sont plus complexes. En schématisant, on peut dire que les modèles de Masters et Johnson prenaient en considération uniquement la phase d'excitation sexuelle (arousal) et l'orgasme, et qu'Helen Kaplan (7) avait ajouté à ce modèle le désir. Althof (1) présente un modèle d'une complexité adéquate pour prendre en compte les dimensions personnelles, contextuelles, et interpersonnelles du dysfonctionnement sexuel. La dimension personnelle comprend non seulement les niveaux de l'orgasme, de l'excitation et du désir, mais au dessus d'eux ceux de l'identité de genre, du choix d'objet et de l'intention sexuelle : la dimension contextuelle est, comme son nom l'indique, celle des circonstances extérieures et des événements de vie ; la dimension interpersonnelle est plus particulièrement celle de l'équilibre sexuel du couple, soulignant que le dysfonctionnement de l'un des membres retentit obligatoirement sur l'autre. Avec un tel modèle, la thérapie sexuelle ne peut se réduire à des prescriptions comportementales et nécessite des interventions complexes, qu'elles soient psychodynamiques et relationnelles, ou cognitivocomportementales, ou systémiques, ou éclectiques.

Les indications actuelles des psychothérapies psychodynamiques et relationnelles dans le traitement des troubles sexuels doivent se séparer en indications de psychothérapies non centrées sur le symptôme d'une part, et centrées sur le symptôme d'autre part.

Les psychothérapies psychodynamiques et relationnelles non centrées sur le symptôme sont indiquées en tant que psychothérapies individuelles dans les troubles du désir (10), l'impuissance érectile primaire (1), l'impuissance érectile primaire et l'éjaculation précoce primaire (16). Ces indications se conçoivent assez bien dans la mesure où les troubles cités ne sont pas seulement sexuels mais liés de facon prépondérante à 
une dynamique intra-psychique et/ou relationnelle spécifique au patient. De même, ces psychothérapies non centrées sur le symptôme sont indiquées pour le couple lorsque les problèmes conjugaux généraux sont prépondérants par rapport aux difficultés purement sexuelles.

Lorsque les psychothérapies brèves psychodynamiques et relationnelles sont indiquées dans des procédures thérapeutiques centrées sur les symptômes sexuels, elles sont en régle associées à des prescriptions et à des interventions cognitivocomportementales. C'est le cas d'à peu près tous les troubles sexuels psychogènes secondaires, succédant à des difficultés circonstancielles, et en général liées à une anxiété de performance et à des problèmes de communication tournant autour de la sexualité. Dans ces cas-là, il s'agit en régle d'indications de traitement de couple, maintenant classiques en sexothérapie.

Qu'il s'agisse de sexothérapie centrée sur le symptôme, ou de psychothérapie non centrée sur lui, je suis d'accord avec Althof pour souligner la spécificité relative de ces indications respectives. Certains troubles sexuels primaires peuvent en effet répondre à des sexothérapies classiques centrées sur le symptôme, tandis que certains troubles secondaires sont tout à fait sensibles à une psychothérapie générale.

Un dernier point doit être abordé à propos de l'utilisation actuelle en sexothérapie des psychothérapies brèves psychodynamiques et relationnelles, c'est celle des psychothérapies dites "armées", c'est-à-dire associées à un traitement médical. Il s'agit d'un problème classique en psychiatrie dont la plupart des spécialistes s'accordent pour le résoudre en pratiquant une co-thérapie : le patient a deux thérapeutes, l'un qui s'occupe des médicaments et des questions pratiques, et l'autre uniquement de la psychothérapie. Ce dispositif sert à éviter la confusion qui peut résulter des réponses différentes données au patient lorsqu'il a un seul thérapeute qui va tantôt réagir à ses messages par une prescription médicamenteuse et tantôt par une remarque psychothérapique. Il a aussi le mérite de souligner que les domaines des prescriptions des traitements biologiques et des traitements psychologiques ne se recouvrent pas et que l'un ne dispense pas de l'autre, ce qui devient de plus en plus évident dans la psychiatrie moderne.

L'association Injection Intracaverneuse (IIC) /psychothérapies psychodynamiques et relationnelles est une véritable nouveauté. Il n'y a pas de raison de la rejeter à priori, puisque l'on associe bien diverses techniques psychothérapiques en sexologie, et que dans d'autres domaines une psychothérapie peut être associée au traitement biologique d'une affection psychiatrique ou somatique. Kaplan (8), écrit qu'un abord psychodynamique des problèmes individuels ou de couple peut être associé aux I.I.C pour permettre au sujet et/ou à sa partenaire de les utiliser au mieux, et qu'à l'inverse les I.I.C peuvent être un moyen de surmonter les difficultés du sujet et/ou de sa partenaire à entrer dans un processus sexothérapique psychodynamique. Ces indications sont pour elle exceptionnelles : l'association I.I.C/psychothérapie dans un sens ou dans l'autre, n'est pas le moyen infaillible ni toujours indiqué de forcer avec succès dans leur dernier retranchement les sujets et couples réfractaires au traitement. En accord avec cet auteur, mais dans une formulation sensiblement différente, je prônerais l'accompagnement constant des I.I.C par une action psychothérapique adaptée, explicative et de soutien, avec quelques interventions de type psychodynamique ou relationnel si besoin. $\mathrm{Ce}$ genre d'accompagnement psychothérapique d'une thérapeutique somatique à fort impact émotionnel devrait être la régle en médecine. Il ne pose pas de problème théorique, mais celui, non résolu, de la formation et de la compétence psychothérapique des médecins. Associer secondairement les I.I.C à un traitement psychothérapique des troubles érectiles fait penser à la question des psychothérapies dites "armées". Je vois mal, en cours de psychothérapie, le psychothérapeute proposant de faire lui-même quelques I.I.C. Par contre, j'imagine assez bien que devant la stagnation du processus psychothérapique et après avoir soigneusement pesé sa décision, le thérapeute puisse proposer au patient quelques I.I.C faites par son confrère urologue par exemple. Helen Kaplan (8) ne précise pas si dans ces cas-là elle fait elle-même les I.I.C ou en confie le soin à un collaborateur. Peut-être ma position en faveur de la co-thérapie est-elle un préjugé lié à ma pratique ? Il n'en reste pas moins que les psychothérapies "dopées" par I.I.C devraient probablement rester l'exception plus que devenir la régle.

\section{CONCLUSION}

La validité des psychothérapies psychodynamiques et relationnelles, conformément aux standards médicaux, a été démontrée dans les années 80. Leur champ est différent de celui des traitements biologiques, ce qui peut impliquer une concurrence sans merci, mais aussi et plutôt une complémentarité, comme cela se revèle de plus en plus le cas en psychiatrie. Dans le domaine des troubles sexuels, je serais donc pour que l'on travaille à la complémentarité plutôt qu'à la concurrence. Althof (l) souligne que les indications thérapeutiques sont en premier influencées par les valeurs et les préjugés du thérapeute

et que les données de la recherche ne sont pas encore assez importantes et probantes. En accord avec les données de mon exposé, ceci ne peut qu'encourager à l'ouverture d'esprit et à la recherche, tant dans le domaine spécifique des psychothérapies psychodynamiques et relationnelles appliquées aux troubles sexuels, que dans la collaboration avec les cliniciens et chercheurs d'autres disciplines concernées par ces mêmes troubles.

\section{REFERENCES}

1. Althof S E. Psychogenic impotence : treatment of men and couples, in "Principles and practice of sex therapy". Leiblum S R, Rosen R C. The Guildford Press (New York), 1989.

2. Elkin I, Shea M, Watkins J.J et coll. National Institute of Mental Health Treatment of Depression Collaborative Programm : general effectiness of treatment. Arch. Gen. Psychiatry, 1989, 6, 971-982.

3. Held R.R. Psychotherapie et Psychanalyse. 1 vol., Payot, (Paris), 1968.

4. Gillieron E. Les psychothérapies brèves. 1 vol., Payot (Paris), 1983.

5. Gillieron E. Les psychothérapies brèves. Encycl. Med. Chir. (Paris). Psychiatrie, 37812 L 10, 12-1987, $10 \mathrm{p}$.

6. Greenberg E S, Horvath A 0. Rôle de l'alliance thérapeutique apprécié par la recherche sur les psychothérapies, in "Recherches Cliniques Planifiées sur les Psychothérapies" Gerin P et Dazord A. 1 vol. La Documentation Française, 1992.

7. Kaplan H S. The new sex therapy. 1 vol. Brunner/Mazel (New York), 1974.

8. Kaplan H S. The combined use of sex therapy and intrapenile injections in the treatment of impotence. J. Sex. Marital Ther., 1990, 16, 4, 195-207.

9. Klerman G L, Weissman M M, Rounsaville B C, Chevron E S. Interpersonal psychotherapy of depression. 1 vol. Basics Books (New York), 1984.

10. Rosen R C, Leiblum S R. Assessment and treatment of desire disorders, in "Principles and Pratice of sex therapy". Leiblum S R, Rosen R C., The Guildford Press (New York), 1989. 
11. Lo Piccolo J. Post modern sex therapy for erectile failure, in "Erectile Disorders". Rosen R C and Leiblum S R, 1 vol., The Guildford Press (N.Y), 1992.

12. Luborsky L. Principles of psychanalytic psychotherapy. 1 vol. Basic Books (New York), 1984.

13. Orlinsky D, Howard K. A generic model of psychotherapy. J. Integ. Ecl Psychother., 1987, 6, 6-27.

14. Orlinsky D E. Apport de la recherche à la connaissance des psychothérapies in "Recherches planifiées sur les psychothérapies" Gerin P et Dazord A. 1 vol. La Documentation Française, 1992.

15. Masters W, Johnson V. Human Sexual Inadequacy. 1 vol. Little Brown (Boston), 1970.

16. Tignol J. Les Troubles Sexuels Masculins. 1 vol. Masson (Paris), 1991.
RESUME : Les psychothérapies brèves psychodynamiques et relationnelles sont des techniques psychothérapiques réglées inspirées de la psychanalyse ou proches d'elle. Elles se sont surtout développées en Angleterre et aux U.S.A. L'utilisation de procédures standardisées dans leur mise en oeuvre a permis de recueillir à leur sujet dans les années 80 des données fiables prouvant leur efficacité générale, indépendante de la spécificité du modèle psychothérapique choisi. D'où l'élaboration de modèles génériques de psychothérapie valables pour les diverses méthodes, et la possibilité d'en faire un usage éclectique. Dans le traitement des troubles sexuels, les psychothérapies psychodynamiques et relationnelles actuelles prennent en compte, de facon plus importante que ne le faisaient les modèles sexothérapiques inspirés de MASTERS et JOHNSON, la complexité des facteurs intrapsychiques et des problèmes relationnels impliqués dans les dysfonctionnements sexuels. On peut en distinguer les indications centrées sur le symptôme (essentiellement traitement de couple dans les troubles sexuels secondaires), et non centrées sur le symptôme (essentiellement traitement individuel dans les troubles sexuels primaires). La spécificité de ces indications reste toutefois relative. Par ailleurs, l'association psychothérapie/injections intra-caverneuses se discute. Les psychothérapies psychodynamiques et relationnelles ont démontré leur validité. Elles doivent être utilisées dans le champ des troubles sexuels de façon complémentaire, et non concurrentielle, aux traitements biologiques, et continuer à faire l'objet de recherches scientifiques. Mots clés : Psychothérapie brève, Thérapie psychodynamique, Thérapie interpersonnelle, Troubles sexuels. Andrologie, 1992, 2,137 . 140.

\section{REUNIONS DU COURS DE SEXOLOGIE - HOPITAL NECKER Dr R. et Mme C Gellman}

- Mardi 19 Octobre 1993 à 20 h 30 - Le choix conjugal névrotique.

- Mardi 16 Novembre 1993 à 20 h 30 - La sexualité du jeune couple et ses difficultés.

- Mardi 14 Décembre 1993 à 20 h 30 - La jalousie dans le couple.

- Mardi 4 janvier 1994 à 20 h 30 - Les unions transgénérationnelles.

- Mardi 1er Février 1994 à 20 h 30 - Paraphilies et couples.

- Mardi 1er Mars 1994 à 20 h 30 - La Bigamie

- Mardi 29 Mars 1994 à 20 h 30 - La relation extra-conjugale

- Mardi 26 Avril 1994 à 20 h 30 - La contrainte, le conflit, la rupture

- Mardi 17 Mai 1994 à 20 h 30 - Prise en charge thérapeutique des difficultés sexuelles et relationnelles dans le couple.

\section{L'enseignement 1993 - 1994 comprend :}

Un enseignement théorique comprenant 9 soirées réparties au cours de l'année universitaire, le mardi à $20 \mathrm{~h} 30$, et qui ont lieu à l'hôpital NECKER.

Un enseignement semiologique (lère et 2ème années)

Trois journées (un samedi par trimestre de 9 à 12 h et de 14 à 17 h) pour les 3ème année)

Un stage hospitalier (10 matinées dans un service hospitalier) en 3ème année

Des séminaires pratiques sur les thérapies en sexologie ( 3 par an)

Le séminaire de l'AIHUS 3ème week end de mars 94, pour les 3 années. 